\title{
Terrasses climatiques ou terrasses eustatiques pleistocènes le long des cours d'eau de Bretagne occidentale
}

\section{Bernard Hallégouët, Marie Thérèse Morzadec-Kerfourn}

\begin{abstract}
Abstract. - The study of the fluviatile deposits remaining in the valleys of Western Brittany has shown that they generally must be interpreted as climatic terraces related to cold periods. Eustatic interglacial fluviatile terraces seem to have been poorly developed and are apparently not preserved, so that only some old estuarine eustatic sediments have been found.
\end{abstract}

\section{Résumé}

Résumé. - L'examen des dépôts fluviatiles conservés dans les vallées de Bretagne Occidentale a montré qu'ils correspondent pour la plupart à des terrasses climatiques froides. Les terrasses eustatiques fluviatiles interglaciaires, sans doute peu développées à l'origine, ne semblent pas s'être conservées, et pour l'instant seuls de dépôts fluvio-estuariens ont été reconnus.

\section{Citer ce document / Cite this document :}

Hallégouët Bernard, Morzadec-Kerfourn Marie Thérèse. Terrasses climatiques ou terrasses eustatiques pleistocènes le long des cours d'eau de Bretagne occidentale. In: Bulletin de l'Association de géographes français, N441-442, 54e année, Marsavril 1977. pp. 81-89;

doi : https://doi.org/10.3406/bagf.1977.4951

https://www.persee.fr/doc/bagf_0004-5322_1977_num_54_441_4951

Fichier pdf généré le 25/04/2018 
Bull. Assoc. Géogr. Franç., Paris 1977, № 441

\title{
Bernard HALLEGOUET *, Marie-Thérèse MORZADEC-KERFOURN * * \\ TERRASSES CLIMATIQUES \\ OU TERRASSES EUSTATIQUES PLEISTOCENES LE LONG DES COURS D'EAU DE BRETAGNE OCCIDENTALE
}

\begin{abstract}
RESUME. - L'examen des dépôts fluviatiles conservés dans les vallées de Bretagne Occidentale a montré qu'ils correspondent pour la plupart à des terrasses climatiques froides. Les terrasses eustatiques fluviatiles interglaciaires, sans doute peu développées à l'origine, ne semblent pas s'être conservées, et pour l'instant seuls de dépóts fluvio-estuariens ont été reconnus.
\end{abstract}

ABSTRACT. - The study of the fluviatile deposits remaining in the valleys of Western Brittany has shown that they generally must be interpreted as climatic terraces related to cold periods. Eustatic interglacial fluviatile terraces seem to have been poorly developed and are apparently not preserved, so that only some old estuarine eustatic sediments have been found.

\section{POSITION DU PROBLEME}

Les problèmes liés à la genèse des terrasses fluviatiles et à leur âge ont été déjà plusieurs fois étudiés par divers auteurs. Au début du siècle, le Général de Lamothe et Ch. Dépéret attribuaient aux terrasses une origine eustatique. Ils mettaient les hauts niveaux marins correspondants en rapport avec des glaciations et l'impossibilité de leurs conceptions fut par la suite démontrée. On mit alors l'accent sur des causes d'origine climatique ou tectonique.

L'évolution des terrasses climatiques est liée à une surcharge des cours d'eau durant les périodes froides du Pléistocène. Durant ces périodes, les apports abondants provoquent la formation d'une nappe alluviale qui pourra être ultérieurement entaillée par les rivières du fait de la réduction de leur charge. Dans l'Est du Bassin de Paris, (18, p. 379) J. Tricart a montré que des remblaiements puissants se sont constitués lors des périodes froides, dans la partie amont des vallées. Les terrasses des cours d'eau de la façade atlantique de la France

\footnotetext{
- Université de Bretagne Occidentale, E.R.A. 345 du C.N.R.S., B.P. 860, 29279 Brest.

* Université de Rennes, Institut de Géologie et Equipe de Recherche du C.N.R.S. : - Antropologie, Préhistoire, Protohistoire et Quaternaire Armoricains », B.P. 25 A, 35031 Rennes Cedex.
} 
sont aussi en grande partie considérées comme étant d'origine climatique froide $(3,7,8,14,17)$. Dans le Massif Armoricain et ses abords en particulier, les études consacrées aux terrasses de l'Huisne (13), de l'Oust (4) et de la Loire (3) ont montré qu'il s'agissait également de terrasses climatiques. Les alluvions de ces rivières ont été entaillées par l'érosion régressive à la fin de la dernière glaciation, et, dans l'estuaire de la Loire, elles s'amincissent progressivement d'amont en aval pour disparaitre à Mindin (16). Toutefois, dans la vallée sousmarine du Blavet, des accumulations grossières à blocs démesurés ont été reconnues jusqu'à $141 \mathrm{~m}$ sous le niveau actuel (12, p. 213).

Dans sa thèse (18, pp. 286 et 310), J. Tricart estime que, sur la Seine, le creusement préflandrien n'a même pas atteint Paris et que les nappes interglaciaires en pente faible sont rapidement rattrapées en amont par le talweg de creusement qui les entaille. Aussi le développement des terrasses eustatiques ne peut avoir dans un bassin fluvial qu'une extension limitée. Cependant l'exemple des terrasses du Rio Elqui au Chili a permis à R. Paskoff $(11, p$. 183) de montrer qu'un même niveau ancien d'un fleuve peut se raccorder à l'amont à un front morainique et à l'aval à un haut stationnement marin fini-glaciaire, et que cette forme alluviale n'a ni le même âge, ni la même genèse tout au long de son développement.

Les problèmes restent donc ouverts et nous les avons réexaminés à la lumière de recherches sur les cours d'eau de Bretagne Occidentale. Les dépôts superficiels conservés dans leurs vallées n'étaient connus jusqu'ici que d'une manière ponctuelle. Mais une cartographie rigoureuse de leur extension, ainsi que des analyses sédimentologiques et palynologiques, nous ont permis de préciser les conditions de leur mise en place et pour certains d'entre eux leur position stratigraphique.

\section{LES TERRASSES CLIMATIQUES}

\section{Les basses terrasses}

Les basses terrasses de l'Aulne, de l'Elorn et de l'Odet se caractérisent par la fraîcheur de leur matériel et l'abondance des blocs démesurés. Vers l'aval, elles plongent fortement sous les dépôts de la transgression flandrienne. Celle de l'Aulne, à peine entaillée par l'érosion à Châteauneuf du Faou, ne s'élève pas à plus de 8 mètres au-dessus du fond de la vallée. Vers l'aval, elle disparaît dès Châteaulin sous le remblaiement holocène. Celle de l'Odet qui passe sous la vasière actuelle de Kerogan a été reconnue dans l'estuaire. jusqu'à l'entrée de l'anse de Toulven.

Sur l'Elorn, de nouvelles études stratigraphiques et sédimentologiques des basses terrasses nous ont amené à modifier nos premières interprétations au sujet de leur genèse (5). La base des dépôts, dont le matériel est généralement grossier et hétérométrique, s'est mise en place sous un climat périglaciaire humide. Leur partie supérieure, constituée de sables fins bien triés passant progressivement à des 
limons, serait liée à des climats périglaciaires plus secs, plutôt qu'à des causes eustatiques. La présence de rares grains de glauconie dans ces dépôts s'explique par le fait qu'ils remanient des formations estuariennes anciennes où ce minéral est abondant. La basse terrasse de l'Elorn, située vers une quinzaine de mètres d'altitude relative à l'Est de Landerneau, semble disparaitre totalement à l'Ouest de cette ville. En fait, il est possible que les formations à galets rencontrées au cours de sondages, sous les bancs de l'estuaire de l'Elorn (1), en soient le prolongement vers l'aval.

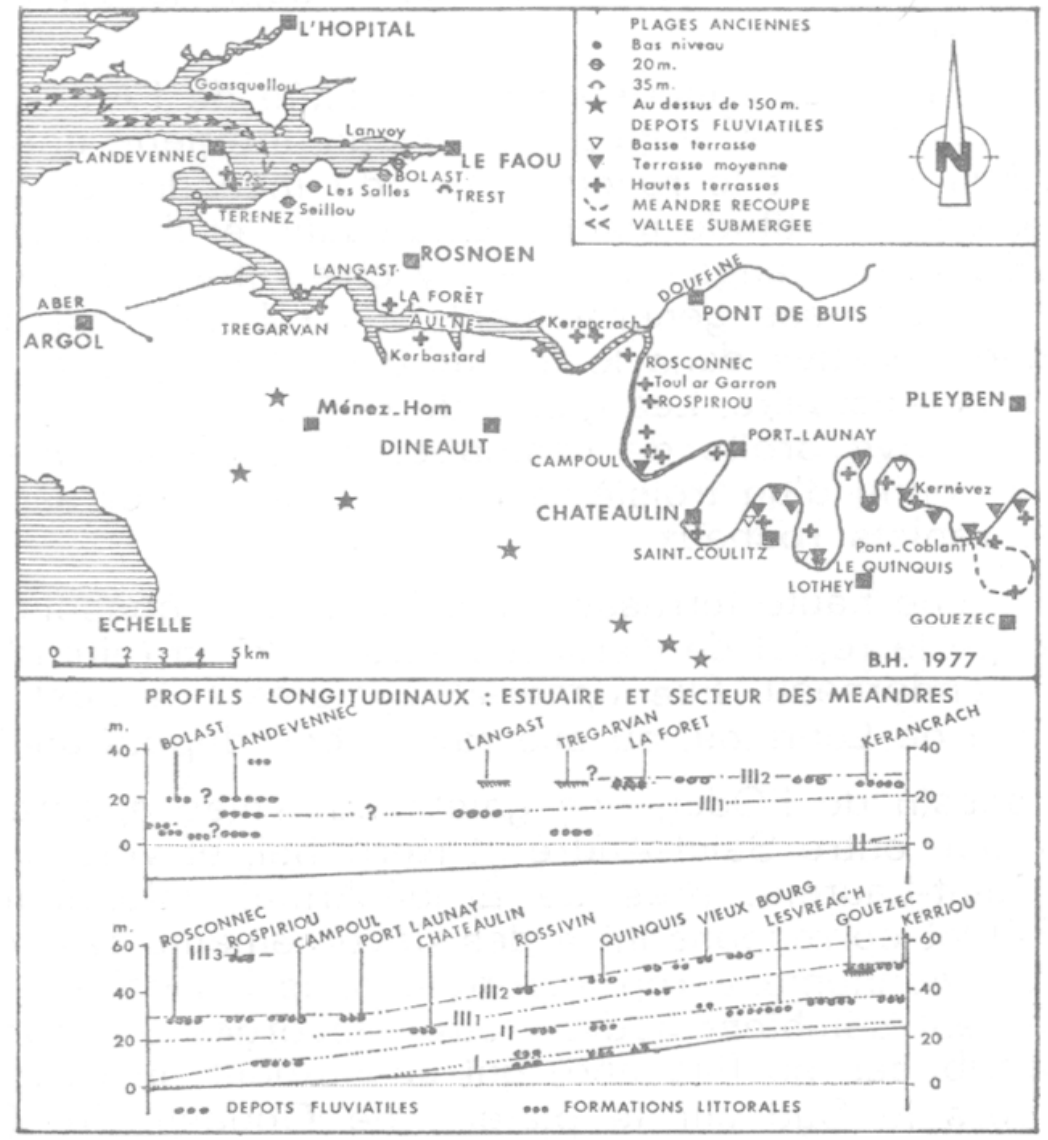

Fig. 1. - La basse vallée de l'Aulne : localisation des dépóts et profils longitudinaux.

Sur l'Aber Ildut, ancienne vallée de l'Aulne-Elorn avant de détournement vers le goulet (5), il n'y a pas à proprement parler de terrasse, mais l'analyse palynologique d'un niveau organique interstratifié dans la nappe alluviale supérieure à PontCorf (6), montre qu'il s'agit d'un dépôt d'origine lacustre. Il renferme les témoins d'une végétation très ouverte à caractère boréal : Herbacées dominantes (Graminées, Ericacées, Polygonacées, Composées), peu d'arbres (Pinus, Betula, rares Picea et Carpinus). Cette association végétale est comparable à celle mise en évidence dans les argiles interstratifiés dans les limons périglaciaires à Port-Lazo en Plouézec (Côtes du Nord) (9). Ces dépôts datent des premiers interstades Weichseliens : AmersfoortBrörup. La coupe de Pont-Corf montre donc qu'une partie des nappes fluviatiles des vallées, s'est mise en place au début du refroidissement Weichselien. 


\section{Les terrasses moyennes et supérieures}

Dans la vallée de l'Aulne, des dépôts formant une terrasse moyenne s'observent dans la région de Châteauneuf-du-Faou vers $25 \mathrm{~m}$ d'altitude relative. Leur altitude décroit progressivement jusquau méandre de Campoul où ils passent sous les vases de l'estuaire. Une terrasse s'élevant au maximum vers $20 \mathrm{~m}$ d'altitude relative apparait également dans le bassin de l'Odet. Elle a été suivie vers l'aval de façon discontinue jusqu'à l'anse de Pors-Guen. Ces terrasses n'admettent pas de blocs démesurés, mais leur plongement vers l'aval indique qu'elles se raccordent à un niveau déprimé correspondant à une période froide.

Les dépôts alluviaux supérieurs de la vallée de l'Aulne se décomposent en trois niveaux étagés. Le niveau inférieur correspond au cours de l'Aulne avant le recoupement du méandre de Gouézec. II a été repéré avec certitude jusqu'à Châteaulin et il semble que, vers l'aval, les dépôts de Langast puissent y être rattachés. Au-dessus se développe une terrasse généralement très rubéfiée, se situant vers $35 \mathrm{~m}$ d'altitude relative dans le méandre du Quinquis et s'abaissant vers $25 \mathrm{~m}$ dans l'estuaire. Les galets de Rospiriou vers $53 \mathrm{~m}$ (altitude N.G.F.), sur la rive droite de l'estuaire, constituent l'unique témoin actuellement connu d'un troisième niveau, auquel correspondent par ailleurs des replats rocheux.

Sur l'Elorn, une haute terrasse rubéfiée, parfois consolidée en poudingue, occupe le replat de Kerliézec vers 30-35 m (altitude N.G.F.) à l'Ouest de Landerneau. Elle apparaît également à l'Est de la ville dans la coupe de Lanrinou. où elle ravine des dépôts anciens.

Dans le bassin de l'Odet, des galets fluviatiles apparaissant vers $110 \mathrm{~m}$ d'altitude entre Saint-André et Kerdohal, et vers $65 \mathrm{~m}$ au Sud du stade Saint-Laurent, près de Ergué-Armel, jalonnent d'anciens tracés de l'Odet correspondant à des déversements temporaires à la suite d'un alluvionnement trop important dans les vallées. Les galets de Saint-Laurent semblent pouvoir être raccordés aux formations de Kernoter (attribuées au Pliocène) qui constituent un delta débordant largement vers le Sud sur le plateau de Gouesnac'h. Ces dépôts représentent également une sédimentation détritique abondante résultant d'une dégradation du climat qui se refroidit au cours du Pliocène et devient plus humide avec des saisons pluvieuses marquées (2).

Les hautes terrasses de l'Aulne et de l'Elorn appartiennent au Pléistocène. Elles n'admettent pas de blocs démesurés, mais les caractères de leur matériel suggèrent une origine climatique. On constate pourtant dans le cas de l'Aulne que leur pente diminue vers l'aval et ne s'accentue pas à l'inverse des terrasses basses et moyennes. On peut donc se demander, du fait de leur concordance altimétrique avec les cordons littoraux anciens du fond de la rade de Brest, si leur édification n'a pas été aussi influencée, pour le cours inférieur de l'Aulne, par des causes eustatiques. 


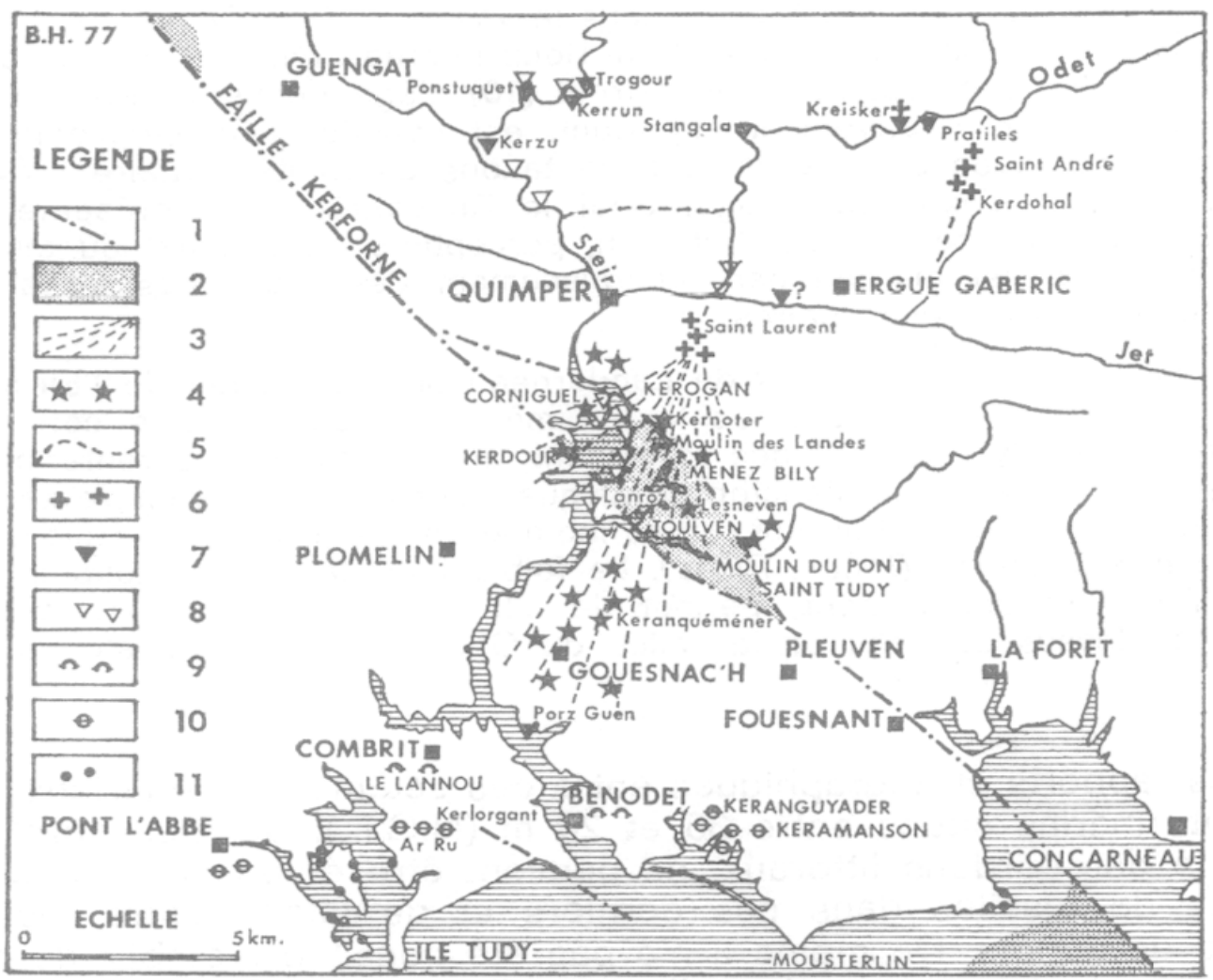

Fig. 2. - Le bassin de l'Odet : localisation des dépóts. - 1. Faille. 2. Formations éocènes. - 3. Ancien delta de l'Odet. - 4. Formations deltaiques et estuariennes. - 5. Anciens tracés hydrographiques. 6. Haute terrasse et nappes alluviales très anciennes. - 7. Terrasse moyenne. - 8. Basses terrasses. - 9. Cordon littoral d'altitude supérieure à $20 \mathrm{~m}$ (N.G.F.). - 10. Plage ancienne située entre 10 et $20 \mathrm{~m}$ (N.G.F.). - 11. Bas niveaux.

\section{LES DEPOTS FLUVIO-ESTUARIENS A CARACTERE EUSTATIQUE}

Le rôle de l'eustatisme semble peu important dans l'édification des terrasses des cours d'eau de la façade atlantique de la France. En Vendée, d'après une analyse pollinique, il apparait pourtant que le remblaiement de la basse terrasse du Lay pourrait être interglaciaire (15, p. 262).

En Bretagne Occidentale le remblaiement eustatique flandrien, peu développé, ne dépasse pas La Roche-Maurice dans la vallée de l'Elorn, et le méandre du Quinquis dans celle de l'Aulne. Les dépôts anciens étudiés pouvant être considérés comme eustatiques, présentent généralement des caractères deltaïques, c'est-à-dire de cours très inférieurs. Ainsi les formations affleurant au Sud de Quimper sont deltaĩques et correspondent à un niveau marin s'élevant vers 50-55 m. Elles sont fondamentalement différentes des dépôts littoraux situés à l'embouchure de l'Odet, qui se caractérisent par une forte éolisation et des apports de minéraux étrangers au bassin de l'Odet. 
Des dépôts estuariens s'étagent dans la vallée du Guindy (Côtes-du-Nord) entre $55 \mathrm{~m}$ à La Salle et $35 \mathrm{~m}$ à Pont-Rouz (altitude (N.G.F.). Ils renferment une végétation à caractère tempéré-froid (10) d'âge Reuverien-Prétiglien. Le couvert forestier, relativement important, est constitué essentiellement par des conifères avec une persistance de taxons à affinité tertiaire : Sciadopitys, Sequoia, Taxodium, Tsuga. Seul le Reuverien est représenté à La Salle. Le refroidissement du Prétiglien, perceptible au sommet du gisement de Pont-Rouz, est marqué par l'accroissement des graminées et la chute brutale des essences tertiaires.

Le dépôt de Lanrinou, près de Landerneau dans la vallée de l'Elorn, culmine à $20 \mathrm{~m}$ (N.G.F.) et présente les mêmes caractères que ceux de la vallée du Guindy. Ce sont des formations argilo-graveleuses renfermant de la glauconie, ainsi qu'un plancton organique marin, mais à caractère saumâtre plus accusé. La végétation conservée indique un climat tempéré-froid : dominance des conifères (Pinus, Picea). La faible importance des taxons tertiajres (Tsuga essentiellement) permet de situer ce gisement chronologiquement au-dessus de celui de la vallée du Guindy, dans le Pléistocène inférieur.

Les rapports stratigraphiques entre ces sédiments estuariens et les dépôts marins situés entre 35 et 20 m (N.G.F.), qui eux correspondent à des cordons littoraux, ne peuvent être établis du fait de l'ab sence de fossiles dans ces derniers et de jalons entre les deux types de dépôts. De même il n'a pas été possible d'établir de corrélations entre ceux-ci et les alluvions fluviatiles affleurant en amont dans les vallées. La plupart d'entre elles, d'origine climatique, plongent en effet vers l'aval sous les alluvions récentes. Même dans le cas des hautes terrasses de l'estuaire de l'Aulne, qui semblent se raccorder à des niveaux constants, le raccord avec les plages anciennes du fond de la rade de Brest n'a pu être effectué.

\section{CONCLUSION}

L'examen des dépôts superficiels conservés dans les vallées des cours d'eau de Bretagne Occidentale a montré que les sédiments qui les ont colmatées se composent d'alluvions fluviatiles et de formations fluvio-estuariennes ou deltaĩques. Ces dépôts entaillés ensuite par l'érosion ont formé des terrasses. La vallée de l'Aber lldut, par où s'écoulaient les cours d'eau de la rade de Brest, présente un cas particulier, car les dépôts qui y sont conservés sont actuellement perchés au-dessus de la vallée de l'Elorn, mais n'ont pas encore été entaillés par l'érosion régressive de l'lldut.

La plupart des terrasses reconnues sont d'origine climatique froide. Les dèpôts se sont mis en place sous des climats périglaciaires humides, mais, dans certains cas, ils peuvent correspondre à des climats périglaciaires plus secs. Les terrasses inférieures, qui sont les plus récentes, plongent fortement vers l'aval, tandis que les terrasses supérieures, plus anciennes, présentent souvent une pente faible dans les 


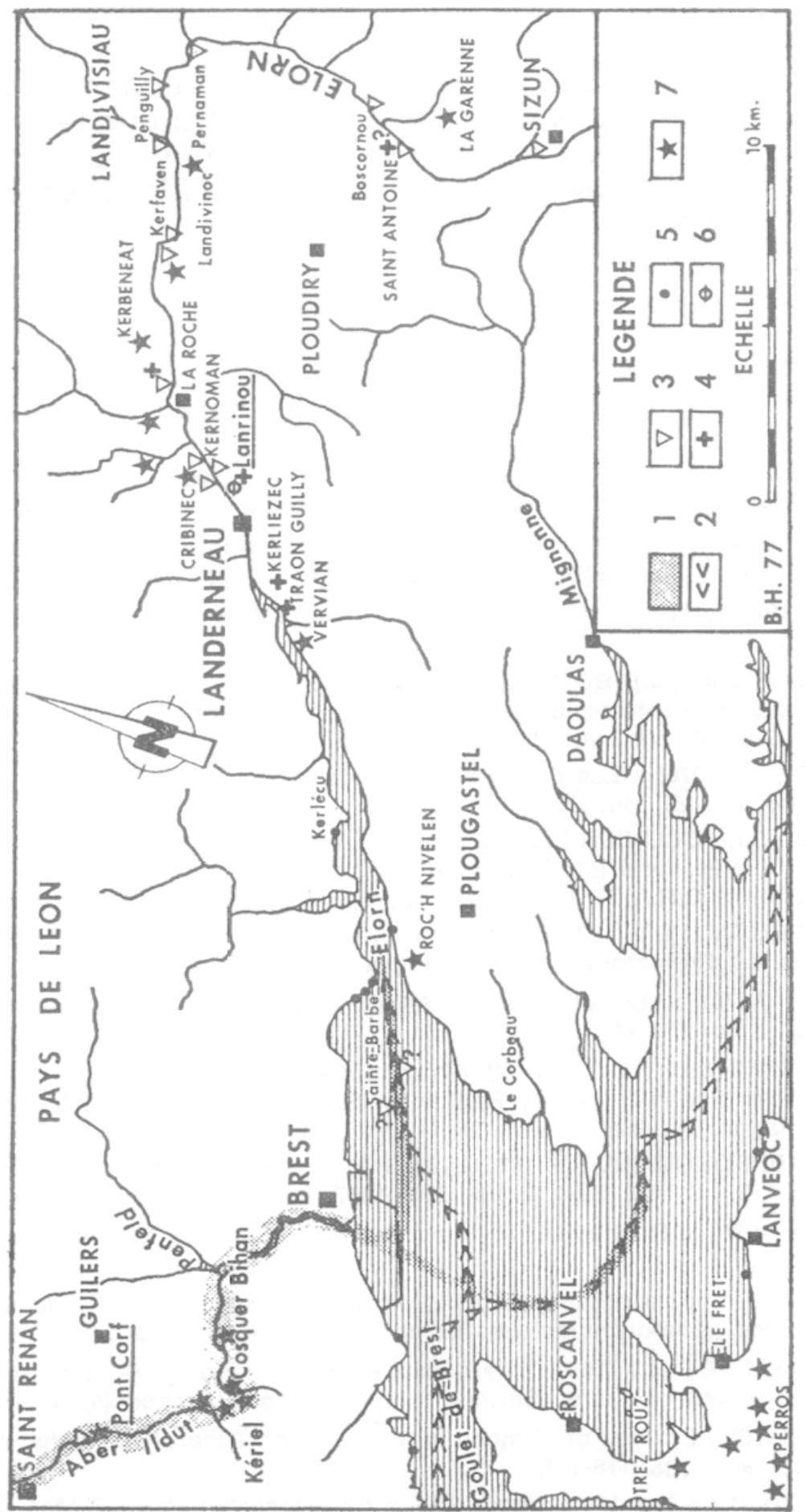

Fig. 3. - Elorn et Aber lldut : localisation des dépóts - 1. Ancien réseau hydrographique. - 2. Vallée submergée. - Dépóts fluviatiles. - 3. Basses terrasses. 4. Hautes terrasses. - Dépots marins et estuariens. 5. Bas niveaux. - 6. Entre 10 et $20 \mathrm{~m}$ (N.G.F.). 7. Au-dessus de $20 \mathrm{~m}$. 
estuaires et pourraient correspondre altimétriquement à des niveaux moins élevés.

Aucune terrasse fluviatile n'a été identifiée comme eustatique et interglaciaire. Les analyses effectuées ont montré que les dépôts interglaciaires ne comprenaient que des sédiments fluvio-estuariens. Ceuxci marquent dans les vallées du Guindy et de l'Elorn les étapes de la régression pliocène.

\section{REFERENCES BIBLIOGRAPHIQUES}

1. BABIN (C.), DIDIER (J.), MOIGN (A.), PLUSQUELLEC (Y.), (1969) : Goulet et rade de Brest ; essal de géologie sous-marine. Rev. Géogr. Phys. Géol. Dyn., XI, 1, pp. 54-64.

2. ESTEOULE-CHOUX (J.) (1970) : Contribution à l'étude des argiles du Massif Armoricain. Thèse Rennes, Mém. Soc. Géol. Min. Bretagne, 14, 319 p.

3. GRUET (M.) (1976) : Les alluvions fluviatiles dans le Bassin de la Loire. La Préhistoire française, 1, pp. 112-114.

4. GUILCHER (A.), TERS (M.), BONNET (J.-Y.) (1961) : Données récentes sur l'évolution morphologique des vallées bretonnes : Oust et vallée de .Saint-Renan. Comité Trav. Hist. et Scientif., Bull. Sect. Géogr., vol. 74, pp. 1-53.

5. HALLEGOUET (B.) (1976) : Les anciens dépôts marins et fluviatiles de la vallée de l'Elorn (Finistere). Norois, t. 23 , pp. 55-72.

6. HALLEGOUET (B.), OLLIVIER-PIERRE (M.-F.), ESTEOULE-CHOUX (J.) (1976) : Découverte d'un dépót oligocène inférieur dans la haute vallée de l'Aber lldut, au Nord-Ouest de Brest (Finistère), C.R.A.S., t. 283, Sér. D., pp. 1711-1714, Paris.

7. ICOLE (M.), RIEUCAU (L.) (1976) : Les alluvions fluviatiles dans le bassin de la Garonne et ses affluents. La Préhistoire française, I, pp. 101-104.

8. MICHEL (J.-P.) (1976) : Les alluvions fluviatiles dans le Bassin de la Seine. La Préhistoire francaise, I, pp. 115-117.

9. MORZADEC-KERFOURN (M.-T.) (1974) : Variations de la ligne de rivage armoricaine au Quaternaire. Thèse Rennes, Analyse Pollinique de dépóts organiques littoraux. Mém. Soc. Géol. Min. Bretagne, 17, 208 p.

10. MORZADEC-KERFOURN (M.-T.) (1975) : Le Pllo-Quaternaire marin de Pont-Rouz (Cótesdu-Nord). C.R.A.S., t. 280, Sér. D, pp. 1955-1957, Paris.

1. PASKOFF (R.) (1970) : Recherches géomorphologiques dans le Chili semi-aride. Thèse, Bordeaux, $420 \mathrm{p}$.

12. PINOT (J.-P.) (1974) : Le précontinent breton entre Penmarc'h, Belle-lle et l'escarpement continental : Etude géomorphologique. Thèse Brest, Lannion, 256 p.

13. POSER (H.), TRICART (J.) (1950) : Terrasses et phénoménes périglaciaires dans la vallée de l'Huisne en amont du Mans (Sarthe). Sull. Soc. Géol. France (5) XX, pp. 381-391.

14. SOMME (J.) (1976) : Formes et formations fluviatiles en Picardie et dans le Nord. La Préhistoire française, I, pp. 118-121.

15. TERS (M.) (1961) : La Vendée littorale. Etude de Géomorphologie. Thèse, 580 p., Rennes.

16. TERS (M.), PLANCHAIS (N.), AZEMA (C) (1968) : L'évolution de la Basse vallée de la Loire à l'aval de Nantes à la fin du Würm et pendant la régression flandrienne. Bull. A.F.E.Q., 5, pp. 217-246, Paris.

17. TEXIER (J.-P.), THIBAULT (C.) (1976) : Les alluvions fluviatiles dans le Sud-Ouest. La Préhistoire française, I, pp. 107-111.

18. TRICART (J.) (1952) : La partie orientale du Bassin de Paris. Thèse Paris, 471 p. 


\section{DISCUSSION}

VIERS : Les hautes terrasses arrivent à la mer bien au-dessus du niveau actuel ; mais les basses terrasses plongent sous le remblaiement flandrien. Les basses terrasses étant Riss ot Würm, les hautes peuvent être du Pléistocène inférieur, mais, si elles sont périglaciaires à l'amont, pourquol ne se raccordent-elles pas à l'aval à un bas niveau marin? HALLEGOUET : C'est un point sur lequel la discussion reste ouverte.

NICOD : La capture de l'Aulne par la rivière du Goulet est-elle due à l'érosion régressive ou à un mouvement tectonique? HALLEGOUET : Aux deux, car il semble y avoir eu un rejeu du décrochement Kerforne.

PHLIPPONNEAU : Quels sont les grands ancêtres qui ont déjà décrit ce système de capture? HALLEGOUET : Musset, Vacher et Collin.

PHLIPPONNEAU : Vos recherches ont-elles changé quelque chose à leurs conclusions? HALLEGOUET : Oui, car certains de leurs tracés ne peuvent pas être retenus.

DEMANGEOT (note écrite) : II ne faut pas oublier, quand on traite de terrasses climatiques, les travaux de $H$. Baulig, de J. Bourcart, de L. Trevisan. 